Pacific Journal of Mathematic 


\title{
ON FINITE-DIMENSIONAL UNIFORM SPACES
}

\author{
J. R. ISBELL
}

Introduction. This paper has two nearly independent parts, concerned respectively with extension of mappings and with dimension in uniform spaces. It is already known that the basic extension theorems of point set topology are valid in part, and only in part, for uniformly continuous functions. The principal contribution added here is an affirmative result to the effect that every finite-dimensional simplicial complex is a uniform ANR, or ANRU. The complex is supposed to carry the uniformity in which a mapping into it is uniformly continuous if and only if its barycentric coordinates are equiuniformly continuous. (This is a metric uniformity.) The conclusion (ANRU) means that whenever this space $\mu A$ is embedded in another uniform space $\mu X$ there exist a uniform neighborhood $U$ of $A$ (an $\varepsilon$-neighborhood with respect to some uniformly continuous pseudometric) and a uniformly continuous retraction $r: \mu U \rightarrow \mu A$.

It is known that the real line is not an ARU. (Definition obvious.) Our principal negative contribution here is the proof that no uniform space homeomorphic with the line is an ARU. This is also an indication of the power of the methods, another indication being provided by the failure to settle the corresponding question for the plane. An ARU has to be uniformly contractible, but it does not have to be uniformly locally an ANRU. (The counter-example is compact metric and is due to Borsuk [2]). It does have to be uniformly locally connected, which is enough to give us a grip on the real line.

Smirnov has defined the $\delta$-dimension $\delta d$ of a uniform space as the least dimension of a cofinal family of finite uniform coverings and has shown that $\delta d$ has many of the properties of topological dimension functions and some novel ones $[9,10]$. The large dimension $\Delta d$ is defined in the same manner, using arbitrary uniform coverings, in [6], where it is shown that $\Delta d$ is $\geqq \delta d$ and is (like $\delta d$ ) invariant under completion. The central result of the second part of this paper is that when $\delta d(\mu X)=n$ there are precisely two possible values for $\Delta d(\mu X)$, namely $n$ and $\infty$.

Two applications are made, the principal one being a considerable simplification of the proof of the main theorem of [10] (characterization of the $n$-dimensional uniform subspaces of $E^{n}$ ). Also there are two side conditions either of which implies $\Delta d(\mu X)=\delta d(\mu X)$ : every uniform covering has a finite-dimensional uniform refinement $(\mu X)$ is, so to speak, weakly finite-dimensional), or $\mu X$ is locally fine in the sense of [5]. The

Received May 26, 1958. 
first of these includes the case of a weak uniformity induced by a family of real-valued functions, and the second includes the case of the finest uniformity compatible with the topology.

1. Extension. Most of the simplicial complexes considered in this paper will be finite-dimensional, largely because we do not have a convenient uniform structure for infinite-dimensional complexes. In any simplicial complex $X$, the points $x$ are determined by their barycentric coordinates $\left(x_{\alpha}\right)$. The function $d(x, y)=\max \left|x_{\alpha}-y_{\alpha}\right|$ is a distance function inducing a uniformity and topology on $X$; with this uniformity, $X$ is called a uniform complex. We recall from [7] that a finite-dimensional uniform complex is a complete space, the stars of vertices from a unifom covering $\left\{S t_{\alpha}\right\}$, a mapping into $X$ is uniformly continuous if and only if its coordinate projections are equiuniformly continuous ; and every finitedimensional uniform covering of a uniform space has an equiuniformly continuous partition of unity subordinated to it, which then induces a canonical mapping into the nerve.

In fact, we can show the following.

1.1. Every uniform covering has an equiuniformly continuous partition of unity subordinated to it.

Because of the difficulty with infinite-dimensional complexes, we shall not get any use out of 1.1 excepting a very special application in the second section of the paper.

Proof of 1.1. Let $\left\{U_{\alpha}\right\}$ be a uniform covering of a uniform space $\mu X$. Let $d$ be a uniformly continuous pseudometric on $\mu X$ such that every set of $d$-diameter 2 or less is contained in some $U_{\alpha}$ [7]. Well order the indices $\alpha$. For each $\alpha$, we define a real-valued function $g_{\alpha}: g_{\alpha}(x)$ is the smaller of the numbers 1 and $\sup \left[d\left(x, X-U_{\beta}\right) \mid \beta<\alpha\right]$. The functions $g_{\alpha}$ increase monotonically to the pointwise limit 1 (continuously at limit ordinals), and each of them is uniformly continuous with respect to $d$, with modulus of continuity $\delta(\varepsilon)=\varepsilon$; that is, $d(x, y)<\varepsilon$ implies $\left|g_{\alpha}(x)-g_{\alpha}(y)\right|<\varepsilon$. Therefore the functions $f_{\alpha}=g_{\alpha+1}-g_{\alpha}$ form an equiuniformly continuous partition of unity; and if $x \in X-U_{\alpha}$, then $g_{\alpha+1}(x)=g_{\alpha}(x)$ and $f_{\alpha}(x)=0$.

A partition of unity $\left\{f_{\alpha}\right\}$ will not yield a function with values in the nerve unless at each point all but finitely many $f_{a}$ vanish. An obvious sufficient condition for this is that $\left\{U_{\alpha}\right\}$ is point-finite. We can rearrange the statement of this condition by using the following (essentially known) construction. A covering $\left\{V_{\alpha}\right\}$ with the same indexing set as $\left\{U_{\alpha}\right\}$ is called a shrinking of $\left\{U_{\alpha}\right\}$ if $V_{\alpha} \subset U_{\alpha}$ for all $\alpha$; let us call $\left\{V_{\alpha}\right\}$ a 
strict shrinking if there exists a uniform covering $w$ such that $\operatorname{St}\left(V_{\alpha}, w\right) \subset U_{\alpha}$ for all $\alpha$.

1.2. Every uniform covering has a strict shrinking which is uniform. If a uniform covering $w$ is a star-refinement (or merely a refinement) of $u$, then $u$ can be strictly shrunk (or merely shrunk) to a uniform covering $v$ whose elements are unions of disjoint families of elements of $w$.

Proof. Well order the elements $U_{\alpha}$ of $u$ and define $V_{\alpha}$ as the union of all elements of $w$ whose stars (or merely whose selves) are contained in $U_{\alpha}$ but not contained in any preceding $U_{\beta}$.

At the moment, we want the following applications of 1.2. Every point-finite uniform covering has a uniformly locally finite uniform refinement (any strict shrinking); and if a covering has a uniformly locally finite refinement then it has a uniformly locally finite shrinking.

Note specifically that the nerve of the covering $v$ constructed in 1.2 is a subcomplex of the nerve of $u$ and is the image of the nerve of $w$ under a simplicial mapping. The dimension of $v$ is no greater than the dimension of $w$.

In normal topological spaces 1.2 is valid for coverings having locally finite refinements, and leads to the conclusion that such a covering admits a canonical mapping into the nerve for any reasonable topology on the nerve [3]; for continuity is easily deduced from the fact that a neighborhood of each point is mapped into a finite complex. A uniform space is called locally fine if every uniformly locally uniform covering is uniform [5]; it follows that every uniformly locally uniformly continuous function is uniformly continuous, and also that every uniform covering has a uniformly locally finite uniform refinement. Accordingly we have

1.3. Relative to any uniformity for simplicial complexes which makes every finite subcomplex a uniform complex, the following is true: Corresponding to every uniform covering of a locally fine uniform space there is a canonical mapping into the nerve.

We shall want to apply this with some structure on the nerve of $\left\{U_{\alpha}\right\}$ making $\left\{S t_{\alpha}\right\}$ a uniform covering. It will suffice to use uniform Whitehead complexes (UW-complexes) defined as CW-complexes bearing the finest uniformity compatible with the topology.

We obtain also, from 1.1 and 1.2, certain mappings of any uniform space into the nerve (regarded as a uniform complex) of any covering having a point-finite uniform refinement. The mappings take $U_{\alpha}$ into $S t_{\alpha}$, but when $\left\{S t_{\alpha}\right\}$ is not uniform, this is of little value. 
1.4. LemMa. Every bounded uniformly continuous pseudometric on a subspace of a uniform space many be extended to a bounded uniformly continuous pseudometric on the whole space.

Proof. Suppose $e$ is a bounded pseudometric on $\mu A \subset \mu X$. We show first that there is a pseudometric $d$ on $\mu X$ satisfying $d(x, y)>e(x, y)$ for all $x$ and $y$ in $A$. For each integer $n$ (positive or negative) there is a uniform covering $u^{n}$ of $\mu X$ such that if $x$ and $y$ are in $A$ and in a common element of $u^{n}$ then $e(x, y)<2^{n}$; and there is a pseudometric $d_{n}$ on $\mu X$ such that $d_{n}(x, y)<2^{n+1}$ implies $x$ and $y$ are in a common element of $u^{n}$, but $d_{n}(x, y) \leq 2^{n+1}$ for all $x$ and $y$. If $2^{k}$ is a bound for values of $e$, then $\sum\left(d_{n} \mid k \geq n>-\infty\right]$ is a pseudometric $d$ uniformly continuous on $\mu X$, and $d(x, y)<2^{n+1}$ implies $e(x, y)<2^{n}$, so that $d>e$ in $A$. Finally define $m$ on $X$ by $m(x, y)=\min (d(x, y)$, inf $[d(x, a)+e(a, b)+d(b, y) \mid a$ and $b$ in $A]$ ). Examination of cases shows that $m$ is a pseudometric. Since $m \leq d, m$ is uniformly continuous. Then $m$ is the required extension of $e$.

1.5. CoRollary. For every uniformly continuous mapping $f$ of a subspace $\mu A$ of a uniform space $\mu X$ into a metric space $\nu B$, there exist $a$ metric space $\nu Y$ containing $\nu B$ and a mapping $g: \mu X \rightarrow \nu Y$ extending $f$.

For every metric is equivalent to a bounded one.

1.6. CoRollary. For every uniformly continuous mapping $f$ of a subspace $\mu A$ of a uniform space $\mu X$ into a uniform space $\nu B$, there exist $a$ uniform space $\nu Y$ containing $\nu B$ and $a$ mapping $g: \mu X \rightarrow \nu Y$ extending $f$.

For every uniform space is a subspace of a product of metric spaces.

The definitions of absolute retract and absolute neighborhood retract write themselves, except that one must notice that uniform neighborhoods should be specified. If $A \subset U \subset \mu X, U$ is a uniform neighborhood of $A$ provided $U$ contains the star of $A$ with respect to some uniform covering. $\mu X$ is an ARU provided $\mu X \subset \mu Y$ implies the existence of a uniformly continuous retraction $\mu Y \rightarrow \mu X ; \mu X$ is an ANRU provided $\mu X \subset \mu Y$ implies the existence of a uniformly continuous retraction onto $\mu X$ of some uniform neighborhood of $X$ in $\mu Y$. One point to be noticed is

1.7. Every ANRU is complete; moreover, an incomplete space can be embedded as a closed subspace of a space in which there is no retraction of a uniform neighborhood.

Proof. Given any incomplete space $\mu A$, let $\pi \mu A$ be its completion 
and $I$ a well-ordered space, in the order topology, having a last element $\omega$ such that every sequence converging to $\omega$ has a greater cardinal number than that of $A$. Embed $\mu A$ in $\pi \mu A \times I, \alpha \in A$ going to $(\alpha, \omega)$, and remove from the product the points $(x, \omega), x$ not in $A$; evidently $\mu A$ becomes a closed subspace on which there is no continuous retraction of a neighborhood.

Among topological spaces one distinguishes between the property of being an absolute retract (or an ANR) and the stronger property of being an absolute (neighborhood) extensor: $Y$ is an absolute extensor for a class of spaces if when $A$ is a closed subspace of a space $X$ in this class, every continuous mapping of $A$ into $Y$ can be extended over $X$. From 1.4 and its corollaries we have the following: Among uniform spaces, every absolute retract is an absolute extensor, i.e. every uniformly continuous mapping of a subspace of any space $\mu X$ into an ARU may be extended. Similarly for ANRU's. Further, for a metric space to be an ARU or ANRU, it suffices that it is a retract or neighborhood retract whenever it is embedded in a metric space. Moreover, if we choose any convenient bounded distance function, we may assume the embedding is an isometry.

The reduction to the isometric case simplifies matters considerably, but there still remains some computation. We shall have to consider moduli of continuity explicitly. Recall that a modulus, in this context, is any function on the positive reals to the positive reals.

1.8. LemMa. For every modulus of continuity $\delta$ and every natural number $n$, there exists a modulus $\lambda$ such that every mapping of a subspace of a metric space into a uniform $n$-simplex, having the modulus $\delta$, can be extended to a mapping of the whole space into the simplex having modulus $\lambda$.

Proof. The $n$-simplex $T$ is an ARU because it is uniformly equivalent to a product of intervals, each of which is an ARU by Katetov's extension theorem [8]. Now suppose the lemma is false, i.e. there exist $\delta$ and $n$ such that for each modulus $\lambda$ there exist metric spaces $A_{\lambda} \subset X_{\lambda}$ (with distance $d_{\lambda}$ ) and mappings $f_{\lambda}: A_{\lambda} \rightarrow T$, each having modulus of continuity $\delta$ but having no extension over $X_{\lambda}$ with modulus $\lambda$. Let $X$ be the union of disjoint copies of all $X_{\lambda}$, with the following distance function $d: d(x, y)=1$ unless $x$ and $y$ are in the same $X_{\lambda}$ and $d_{\lambda}(x, y)<1$, in which case $d(x, y)=d_{\lambda}(x, y)$. Then with $A=\cup A_{\lambda}, f: A \rightarrow T$ defined by the values of the $f_{\lambda}, f$ is uniformly continuous with modulus of continuity $\min (\delta, 1)$. Therefore $f$ has an extension over $X$ which has a modulus of continuity $\lambda$. But $\min (\lambda, 1)$ is a modulus of continuity $\mu$, and the restriction of $f$ to $X_{\mu}$ has modulus $\mu$, a contradiction. 
1.9. THEOREM. Every finite-dimensional uniform complex is an ANRU.

Proof. Suppose the $n$-dimensional complex $N$ is isometrically embedded in $X$ with distance $d$; on $N, d(x, y)=\max \left|x_{\alpha}-y_{\alpha}\right|$. We shall need the product of all the odd numbers up to $2 n+1$; for typographical convenience we take $(2 n+1)$ ! Then let $N_{k}$ denote the $k$-skeleton of $N$, and $C_{k}$ the set of all $x$ in $X$ which satisfy

$$
d\left(x, N_{k}\right) \leq \frac{(2 n+1) !}{(2 k+1) !} d(x, N) \quad \text { and } \quad d\left(x, N_{k}\right) \leq \frac{1}{(2 k+3) !} .
$$

Any $x$ in $C_{0}$ is within distance $1 / 6$ of a unique vertex, which we define to be $f_{0}(x)$; thus $f_{0}: C_{0} \rightarrow N_{0}$ is a retraction with modulus of continuity $\delta \equiv 2 / 3$. Suppose the retraction $f_{k}: C_{k} \rightarrow N_{k}$ has been defined and has a definite modulus of continuity $\delta_{k}$. Now if $x$ is within distance $\theta$ of points $p, q$, in different $(k+1)$-simplexes, $\sigma, \tau$, of $N$, then $p$ and $q$ are within $2 \theta$ of each other. If $(2 k+4) \theta<1$, define barycentric coordinates of a point $r$ by deleting those non-zero coordinates $p_{\alpha}$ of $p$ for which $q_{\alpha}=0$ (whose sum is at most $(k+1) 2 \theta$ ) and increasing accordingly one of the non-zero coordinates of $p$ which must be left. Then $r$ is common to $\sigma$ and $\tau$ and hence is in $N_{k}$; also, $d(x, r) \leq(2 k+3) \theta$. Thus if $x$ is in $C_{k+1}$ and not in $C_{k}$, then there is a unique $(k+1)$-simplex $\sigma$ such that

$$
d(x, \sigma) \leq \frac{(2 n+1) !}{(2 k+3) !} d(x, N) \quad \text { and } \quad d(x, \sigma) \leq \frac{1}{(2 k+5) !} .
$$

Let $C(\sigma)$ denote the union of the set of all such $x$ and $f_{k}^{-1}(\sigma)$; let $A(\sigma)$ denote $\left(C_{k} \cap C(\sigma)\right) \cup \sigma$; and define a retraction $\varphi: A(\sigma) \rightarrow \sigma$ to agree with $f_{k}$ on $C_{k} \cap C(\sigma)$. For pairs of points in $C_{k}, \varphi$ has modulus of continuity $\delta_{k}$; for pairs in $\sigma$, the identity function $\delta(\varepsilon)=\varepsilon$; and for $p$ in $C_{k}, q$ in $\sigma-C_{k}=\sigma^{0}$, we have

$$
d\left(p, N_{k}\right) \leq \frac{(2 n+1) !}{(2 k+1) !} d(p, q),
$$

which yields a point of $N_{k}$ near both $p$ and $q$ and establishes a modulus of continuity here also. By $1.8, f_{k}$ can be extended over each $C(\sigma)$ separately, as a retraction with a definite modulus of continuity $\lambda$. Then $\delta_{k+1}=\lambda /(6 k+12)$ is a modulus of continuity for the whole mapping $f_{k+1}$, since two points at distance $\theta$ from each other in different $C(\sigma)$, $C(\tau)$, are within $(3 k+6) \theta$ of a point $r$ of $\sigma \cap \tau$, as above. Therefore the induction runs, and $f_{n}$ becomes defined on the entire $1 /(2 n+3)$ ! neighborhood of $N$.

It should be noted that the theorem as stated is trivially false for arbitrary uniform complexes, since some of them are incomplete. It is false for many complete ones also. It seems likely that strong results 
might be gotten by using some suitable uniformity for a complex, different from the one defined by $\max \left|x_{\alpha}-y_{\alpha}\right|$, though not necessarily different for finite-dimensional complexes. UW-complexes are different in the finite-dimensional case, and I do not know whether they satisfy 1.9 .

One gets the homotopy extension lemma and the theorem ARU $\equiv$ uniformly contractible ANRU just as in the topological case. Precisely, if $I$ is the interval $[0,1]$, the cylinder over $\mu X$ is the product $\mu X \times I$, and the cone over $\mu X$ is the quotient space of the cylinder obtained by collapsing $\{(x, 1)\}$ to a point. Homotopy and related concepts being defined as usual, we have

1.10. If $\mu A \subset \mu X, \nu Y$ is an ANRU, $h: \mu A \times I \rightarrow \nu Y$ is a homotopy between $h_{0}$ and $h_{1}$, and $g_{0}$ is an extension of $h_{0}$ over $\mu X$, then $h$ can be extended to a homotopy of $g_{0}$.

Proof. Define $f$ on $(\mu A \times I) \cup\{(x, 0)\}$ by $f(a, t)=h(a, t), f(x, 0)=$ $g_{0}(x)$. Let $f^{\prime}$ be an extension of $f$ over a uniform neighborhood $U$, and let $p$ be a uniformly continuous real-valued function on $\mu X \times I$ vanishing outside $U$ and equal to 1 on the domain of $f$. Then $g(x, t)=$ $f^{\prime}(x, t p(x, t))$ defines the required extension.

1.11. A uniform spaces is an ARU if and only if it is a uniformly contractible ANRU.

Proof. An ARU is an ANRU by definition, and retraction of the cone over it defines a uniform contraction. Conversely, every mapping into a uniformly contractible ANRU is homotopic to a constant and therefore extensible.

\subsection{The cone over an ANRU is an ARU.}

Proof. Let $\nu Y$ be an ANRU, $C$ the cone over $\nu Y, \mu A$ a subspace of $\mu X$, and $f: \mu A \rightarrow C$ a uniformly continuous function. The construction of $C$ as a quotient space of $\nu Y \times I$ gives each point of $C$ a second coordinate in $I$, and each point except the vertex $v$ has a first coordinate in $Y$. Let $f_{2}: \mu A \rightarrow I$ be the second coordinate of $f$, which is uniformly continuous. Let $f_{1}: A-f_{2}^{-1}(1) \rightarrow Y$ be the first coordinate of $f$, and note that $f_{1}$ is uniformly continuous on each of the sets $A_{n}=f_{2}^{-1}\left[0,1-2^{-n}\right]$. Let $g_{2}: \mu X \rightarrow I$ be a uniformly continuous extension of $f_{2}$. Let $g_{1}$ be a function with values in $Y$, defined on a subset $U$ of $X$, such that for each $n, U$ contains a uniform neighborhood of $A_{n}$ on which $g_{1}$ is a uniformly continuous extension of $f_{1}$. (The construction of $g_{1}$ requires a 
little care. Define $B_{1}=A_{2}, j_{1}=g_{1} \mid B_{1}$. Inductively let $h_{n}$ be an extension of $j_{n}$ over a uniform neighborhood of $B_{n}$, and $i_{n}$ the restriction of $h_{n}$ to a uniform neighborhood $U_{n}$ of $B_{n}$ which is contained in $g_{2}^{-1}\left[0,1-2^{-n-1}\right] ;$ let $B_{n+1}=A_{n+2} \cup U_{n}$, define $j_{n+1}: B_{n+1} \rightarrow Y$ by the values of $i_{n}$ and $g_{1}$, and continue.) If $U_{n}$ is such a neighborhood of $A_{n}$, there exist uniformly continuous pseudometrics $d_{n}$ on $\mu X$ relative to which $U_{n}$ is an $\varepsilon_{n}$-neighborhood of $A_{n}$; we may assume $d_{n}$ is bounded by 1 and form $d=\sum 2^{-n} d_{n}$, so that relative to $d, U_{n}$ is a $\delta_{n}$-neighborhood of $A_{n}$, for a sequence of positive numbers $\delta_{n}$. Let $g_{0}$ be a monotone decreasing continuous function on $I$ to $I$, vanishing only at 1 , but satisfying $g_{0}\left(1-2^{-n}\right)<\delta_{n+1}$. Now define $g_{2}^{\prime}$ on $\mu X$ to $I$ as follows. For $p$ in $A$, we have $g_{2}^{\prime}(p)=f_{2}(p)$. If $d(p, A)>g_{0}\left(g_{2}(p)\right)$ then $g_{2}^{\prime}(p)=1$. For all other $p$ we have

$$
g_{2}^{\prime}(p)=g_{2}(p)+\frac{d(p, A)}{g_{0}\left(g_{2}(p)\right)}\left(1-g_{2}(p)\right) .
$$

One readily verifies that $g_{2}^{\prime}$ is uniformly continuous. Since $g_{2}^{\prime}$ takes the constant value 1 on the complement of $U$, we may define $g: \mu X \rightarrow C$ by $g(x)=\left(g_{1}(x), g_{2}^{\prime}(x)\right)$ where $g_{2}^{\prime}(x) \neq 1, g(x)=v$ where $g_{2}^{\prime}(x)=1$. Then $g$ is a uniformly continuous extension of $f$.

1.12 shows that many ARU's exist. Also, a product of arbitrarily many ARU's is clearly an ARU. On the other hand, the product of a sequence of copies of the real line is not an ANRU; it is not a retract of any uniform neighborhood in the product of cones over the lines.

In the other direction, we have the following.

\subsection{There is no ARU homeomorphic with the real line.}

Proof. An ARU, and even an ANRU, must be uniformly locally connected; for it can be embedded in a product of metric spaces, and thus in a product of Banach spaces, where retraction of a uniform neighborhood establishes the assertion. Now since the only connected subsets of the line are intervals, a uniformly locally connected structure on the line is either incomplete or uniformly locally compact. In view of 1.7 and 1.11, the proof will be completed when we establish

\subsection{Every uniformly locally compact uniformly contractible space} is compact.

In turn 1.14 will be deduced from

1.15. Every uniformly locally compact space has a basis of star-finite uniform coverings. 
Proof of 1.15. Observe that a uniformly locally compact space has a uniform covering $u$ such that the closures of the stars of elements of $u$ are compact; and the same is true for any refinement of $u$. There is a uniform refinement $v$ which has the property that no proper subfamily of $v$ is a covering of the space. To see this, take a pseudometric $d$ so that every set of $d$-diameter 2 or less is a subset of an element of $u$; choose a maximal family of points $p_{x}$ with mutual distances $\geqq 1 / 2$; and define $V_{\alpha}$ as the set of all points within distance 1 of $p_{\alpha}$ except the other $p_{\beta}$. Now each spherical neighborhood of radius $1 / 4$ is contained in one of the $V_{\alpha}$. If $x$ is a point within distance $1 / 4$ of some $p_{\alpha}, V_{\alpha}$ contains the 1/4- neighborhood of $x$. For any other $x$, there is some $p_{\alpha}$ within distance $1 / 2$ of $x$, and since the $1 / 4$ - sphere about $x$ contains no other $p_{\beta}$ by hopothesis, it is a subset of $V_{\alpha}$. Finally, the covering $\left\{V_{\alpha}\right\}$ must be star-finite since the closures of the stars are compact.

Proof of 1.14. Every uniformly contractible space is finitely chainable in the sense of [1]; that is, for any uniform covering $\left\{U_{\alpha}\right\}$ there exist finitely many indices $\alpha_{1}, \cdots, \alpha_{n}$ and a natural number $m$ such that every $U_{\alpha}$ can be joined to one of the $U_{\alpha_{i}}$ by a chain of $m$ or fewer intersecting sets $U_{\beta}$. (In fact, we may take $n=1$.) If the covering is also star-finite, it is finite. Since a uniformly locally compact space is always complete, we have 1.14, and with it, 1.13 .

It is not true that every ANRU is uniformly locally an ARU; at least, not in the sense that there are arbitrarily fine uniform coverings consisting of ARU's. The trouble is that a subspace which is an ARU must be closed, by 1.7. But Borsuk has exhibited [2] a compact 2dimensional $\mathrm{AR}$ in $E^{2}$ in which no closed 2-dimensional proper subset is an ANR. (For compact spaces, $A R \equiv A R U$ and $A N R \equiv A N R U$, since these spaces can be embedded in cubes.)

The converse is not true either. If $S^{n}$ denotes the finite complex which is the boundary of an $n$-simplex, then the uniform complex which is the separated sum of all $S^{n}$ is uniformly locally an ANRU but not itself an ANRU. With a little more care the same effect can be demonstrated with a metric space which is a discrete sum of ARU's (e.g. $\operatorname{arcs} I_{n}$ in $S^{n}$ coming within distance $1 / n$ of every point of the sphere).

An ARU considered as a topological space is an absolute extensor for paracompact spaces ; and similarly for ANRU's. 1.13 seems to rule out any reasonable converse to the first part of this remark.

2. Dimension. The $\delta$-dimension or uniform dimension $\delta d(\mu X)$ of a uniform space $\mu X$ is defined as the least $n$ such that every finite uniform covering has a (finite) uniform refinement whose nerve is $n$-dimensional ; if there is no such $n$, we write $\delta d(\mu X)=\infty$. (In view of 1.2, it does not matter whether the parenthesis "(finite)" is included or not. If it 
is included, we have the original definition of Smirnov except for irrelevant changes in the concept of equivalence of two spaces-Smirnov speaks of proxımity spaces-and we may quote his results $[9,10]$ freely.) Similarly the large dimension $\Delta d(\mu X)$ is the least $n$ such that every uniform covering has an $n$-dimensional uniform refinement [6]. The inequality $\delta d(\mu X) \leqq \Delta d(\mu X)$ is a trivial consequence of 1.2 .

2.1. A finite-dimensional uniform complex is uniformly equivalent to its first barycentric subdivision, by the identity mapping. The stars of vertices in successive barycentric subdivisions form a basis of uniform coverings.

Proof. The first statement is a consequence of the second. For that, it is well known that the meshes of these coverings approach zero, and it remains to show that each is uniform. It is certainly uniform on a uniform neighborhood of the $O$-skeleton; and the proof may be finished by an induction using the fact that the $(k-1)$-skeleton separates all the $k$-simplexes from each other.

2.2 Lemma. A uniform covering has a finite-dimensional uniform refinement if and only if it has a uniform refinement which is a union of finitely many uniformly discrete subcollections.

Proof. Evidently if a covering $u$ is a union of $n$ subcollections which are uniformly discrete (or even merely collections of disjoint sets) then $u$ has dimension at most $n-1$. For the converse, consider the nerve $N(u)$ as a uniform complex, and let $f$ be a canonical mapping of the space $\mu X$ into $N(u)$. The stars of vertices in the first barycentric subdivision of $N(u)$ form a uniform covering $w$ which is a union of $n+1$ collections of disjoint sets, namely the collections of stars of vertices which are centroids of $i$-dimensional simplexes of $N(u)$, for $i=0, \cdots, n$. If $w^{\prime}$ is a uniform strict shrinking of $w$, then $w^{\prime}$ is a union of $n+1$ uniformly discrete subcollections, and the same is true of $f^{-1}\left(w^{\prime}\right)$, which is a uniform refinement of $u$.

2.3. THEOREM. If $\delta d(\mu X)=n$ then either $\Delta d(\mu X)=n$ or $\mu X$ has $a$ uniform covering which has no finite-dimensional uniform refinement.

Proof. We have observed already that $\Delta d(\mu X) \geqq n$. It remains to show that every finite-dimensional uniform covering $u$ has an $n$-dimensional uniform refinement. By 2.2 we may suppose $u$ is the union of uniformly discrete collections $u^{0}, \cdots, u^{p}$.

Let $U_{i}$ be the union of $u^{i}$. Then $u$ is a refinement of $\left.\} U_{i}\right\}$, which is thus a finite uniform covering. By hypothesis $\left\{U_{i}\right\}$ has an $n$-dimen- 
sional uniform refinement, and therefore (by 1.2) it has an $n$-dimensional uniform shrinking $\left\{V_{i}\right\}$. Let $v^{i}$ be the restriction of $u^{i}$ to the subspace $V_{i}$. Each $v^{i}$ is a disjoint collection covering $V_{i}$; hence their union $v$ is an $n$-dimensional covering of the space which is finer than $u$. To show that $v$ is uniform it suffices to show that each $v^{i}$ is uniform on $V_{i}$ (since $\left\{V_{i}\right\}$ is uniform and finite). But with respect to some uniformly continuous pseudometric, the different elements of $u^{i}$ are at mutual distances $>\varepsilon$, and then in the subspace $V_{i}$ each element of $v^{i}$ is an $\varepsilon$-neigborhood of itself.

2.4. EXAmPLE. There are uniform spaces $\mu X$ for which $\Delta d(\mu X)=\infty$ and $\delta d(\mu X)$ has any desired value. Here is an example homeomorphic with a countable discrete space, and having a basis of star-finite uniform coverings. For the description of the structure of $X$, consider the metric space $K$ which is a union of cells $I^{n}$, each isometric to the unit ball in $E^{n}$, with the distance between two points is different cells defined to be 1. Identify the countable set $X$ with a countable dense subset of $K$, for the purpose of stating: a covering of $X$ is to be uniform on $\mu X$ provided it has a refinement of the form $\left\{U_{i \alpha}\right\}$, where the sets $X_{i}=U_{\alpha} U_{i \alpha}$ are finite in number, and on each $X_{i}$, considered as a subspace of $K$, $\left\{U_{i a}\right\}$ is a uniform covering. One easily sees that this family of coverings satisfies Tukey's axioms and thus defines a uniformity; the associated topology is discrete, since some of the sets $X_{i}$ may be single points (lying in no other $X_{j}$ ).

Every finite covering of $X$ is uniform and may be refined by a finite partition, so that $\delta d(\mu X)=0$. On the other hand, if $\left\{V_{\alpha}\right\}$ is a uniform covering of $K$ which has no finite-dimensional uniform refinement (e.g. the covering consisting of all sets of diameter $<1$ ), then $\left\{V_{\alpha} \cap X\right\}$ is a uniform covering of $\mu X$. If it had a finite-dimensional uniform refinement, we should have $X$ partitioned into sets $X_{1}, \cdots, X_{n}$, each $X_{i}$ covered by a finite-dimensional covering $\left\{U_{i \beta}\right\}$ which is uniform on $X_{i}$ considered as a subspace of $K$. Using 2.2, we may as well assume each $\left\{U_{i \beta}\right\}$ is uniformly discrete. Moreover, we may assume $\left\{U_{i \beta}\right\}$ is a strict shrinking of $\left\{V_{\alpha} \cap X\right\}$, so that for some $\varepsilon>0$ the $\varepsilon$-neighborhood of each $U_{i \beta}$ is contained in some $V_{\alpha}$. If $\varepsilon$ is small enough, any two sets $U_{i \beta}, U_{i \gamma}$, are $3 \varepsilon$ apart. Then the $\varepsilon$-neighborhoods of the sets $U_{i \beta}$ form a finite-dimensional refinement of $\left\{V_{\alpha}\right\}$ and a uniform covering of the $\varepsilon / 2$-neighborhood of a dense subset of $K$, that is, a uniform covering of $K$ : a contradiction.

Let us record the last construction for later use.

2.5. If $u$ is a uniform covering of $\mu X$ and $\left\{V_{i \beta}\right\}$ a uniform covering of $\mu A \subset \mu X$ which is a union of $n$ uniformly discrete families, and a strict 
shrinking of $u$, then there is a uniform covering $\left\{W_{i x}\right\}$ of a uniform neighborhood of $A$ having these two properties and satisfying $W_{i \alpha} \cap A=V_{i \alpha}$.

The proof is just as above except that one must introduce a suitable pseudometric.

Dowker's proof [3] that the same covering dimension is obtained for a normal topological space from its finite, star-finite, or locally finite open coverings goes by way of mappings into spheres and depends on (a) constructing canonical mappings and (b) modifying them to be essential onto each simplex. In uniform spaces, of course, the construction is impossible, since the conclusion is false. This need not mean that useful canonical mappings cannot be constructed. The other part of the construction, the removal of inessentiality, is definitely impossible, even in finite-dimensional spaces. This is easily verified for the subspace of the plane consisting of the vertical line segments $x=n,-1 \leqq y \leqq 0$, and $x=n, 1 / n \leqq y \leqq 1$. In a sense, the construction of 1.15 yielding arbitrarily fine uniform coverings which have no proper subcoverings, is the best one can do in general.

In the case of locally fine uniform spaces, Dowker's argument goes through step by step. One has canonical mappings by 1.3 ; every uniform covering has a uniformly locally finite uniform shrinking; and modifications preserving uniform continuity uniformly locally preserve it in the large. Thus we have

2.6. For locally fine uniform spaces, $\delta$-dimension coincides with large dimension.

Note that the result applies to the topological dimension of non-normal completely regular spaces, provided the definitions are framed in terms of normal coverings; these are the uniform coverings in the finest uniformity compatible with the topology, which is always a locally fine uniformity. [5] Smirnov has established some of the properties of the dimension defined in this way by finite normal coverings; and also, for general uniform spaces, the dimension defined by extension of mappings into spheres is $\delta d[9]$.

2.7. A uniform space which is a finite union of subspaces of large dimension $\leqq n$ has large dimension $\leqq n$.

Proof. The $\delta$-dimensions of the subspaces coincide with the large dimensions; then from the sum theorem for $\delta$-dimension [9] we know $\delta d(\mu X)=n$. From 2.3, $\Delta d(\mu X)$ is $n$ or $\infty$. However, every uniform covering of $\mu X$ may be refined by a union of finitely many finite- 
dimensional uniform coverings of the given subspaces, and hence, as in 2.5 , by a finite-dimensional uniform covering of $\mu X$.

In this manner one can choose finite or infinite coverings according to convenience whenever the large dimension is known to be finite. This occurs for example in questions concerning subspaces of finite-dimensional spaces, such as

2.8. (Smirnov) $A$ subset $S$ of Euclidean space $E^{n}$ has $\delta$-dimension $n$ if and only if there exists $r>0$ such that for every $\varepsilon>0$ there is a solid sphere of radius $r$ in which $S$ forms an $\varepsilon$-net.

Proof. The conditions are sufficient, in view of 2.5, for they imply that any uniform neighborhood of $S$ contains a sphere of radius $r$. On the other hand, suppose the conditions not satisfied; thus for each $r>0$ there is $\varepsilon>0$ such that every $r$-sphere contains a point distant by $\varepsilon$ from $S$. Consider the cell complex $K$ the walls of which are formed by the lattice hyperplanes $x_{i}=p, p$ integral. The first barycentric subdivision $K^{1}$ of $K$, and all successive barycentric subdivisions $K^{m}$, are simplicial complexes, with meshes approaching 0 . Moreover, each is a uniform complex. In particular, on the $(n-1)$ skeleton of $K^{m}$, the stars of vertices form a uniform covering $u$. To see this, observe that $u$ is an open covering on any compact portion of space (say, all $\left|x_{i}\right| \leqq 2$ ), hence has a Lebesgue number there, and every point has a spherical neighborhood of radius 1 on which the restriction of $u$ is congruent to a part of $u$ contained in the specified portion.

To construct a uniform $(n-1)$-dimensional covering of $S$ of arbitrarily small mesh $2 \alpha$, choose $m$ so that the mesh of $K^{m}$ is $\alpha$ or less. Let $\left\{S t_{i}\right\}$ be the covering of the $(n-1)$-skeleton of $K^{m}$ by stars of vertices, and $\theta$ a Lebesgue number for this covering (relative to the $(n-1)$-skeleton). Now there exist, first, $r>0$ such that every $n$-simplex $\sigma$ of $K^{m}$ contains a sphere of radius $r$; therefore, by hypothesis, $\varepsilon>0$ such that each $\sigma$ contains a point distant by $\varepsilon$; finally, if $2 \delta=\min (r, \varepsilon)$, each $\sigma$ contains a point $P_{\sigma}$ distant by $\delta$ from both $S$ and the frontier of $\sigma$. For each vertex $i$ of $K^{m}$, let $U_{i}$ consist of $S t_{i}$ together with all open line segments $\left(p, p_{\sigma}\right)$ such that $p$ is a point of $S t_{i}$ and a boundary point of $\sigma$ (thus $i$ is a vertex of $\sigma$ ). Relatively on the complement of the union of the spheres of radius $\delta$ about all $p_{\sigma}$ (a set which contain $S$ ), $\left\{U_{i}\right\}$ has a Lebesgue number, specifically $\theta \delta^{2} / \alpha^{2}$. To see this, observe that if $x$ is in the frontier of $\sigma$, and $y$ is an interior point of $\sigma$ within $\theta \delta / \alpha$ of $x$, we may construct two similar right triangles in the plane determined by $x, y$, and $p_{\sigma}$, as follows. Drop a perpendicular from $p_{\sigma}$ to the hyperplane of a face of $\sigma$ containing $x$, extend the ray from $p_{\sigma}$ to $y$ until it meets some face of $\sigma$ in a point $q$, and drop a perpendicular from $x$ to the line $q y$. 
A sketch shows that $q$ must be within $\theta$ of $x$ and thus in this case some $U_{i}$ contains both $x$ and $y$. In the case of two interior points $y, z$, of one $n$-simplex $\sigma$, with $d(y, z)<\theta \delta^{2} / \alpha^{2}$ and both $y$ and $z$ distant by $\delta$ from $p_{\sigma}$, draw lines from $p_{\sigma}$ through $y$ and $z$ until they meet faces of $\sigma$, and observe that the distance between these lines measured parallel to $y z$ cannot increase beyond $\theta \delta / \alpha$ before one of the lines hits a face. The remaining case, that $y$ and $z$ are in different $n$-simplexes, $\sigma, \tau$, is similar ; use the facts that $y$ and $z$ are nearer to the $(n-1)$-skeleton than to each other and that $\delta \leqq \alpha / 2$ (since $r \leqq \alpha$ ). Finally a point common to $n+1$ or more sets $U_{i}$ would have to be interior to some $n$-simplex $\sigma$; but projection from $p_{\sigma}$ would give a point common to the corresponding sets $S t_{i}$, which is absurd.

Let us conclude with a few further remarks. As the statement of 2.3 exhibits, we do not need to know that the $\Delta$-dimension is actually finite to know that $\Delta d$ and $\delta d$ are the same. In particular, they are the same in any space whose uniformity is the weak uniformity induced by a family of real-valued functions. I do not know whether $\Delta d$ and $\delta d$ coincide for all metric uniform spaces.

Dowker and Hurewicz have shown [4] that the covering dimension dim for a metrizable space coincides with the sequential dimension $d s$ defined as the least $n$ such that there exists a sequence of locally finite open coverings, each of dimension $\leqq n$, of mesh converging to 0 , each a closure-refinement of the preceding one. (In particular, the theorem shows that $d s$ is a topological invariant, though the concept of mesh converging to 0 is not invariant.) Examination of their proof shows that one can replace the closure-refinements by star-refinements, and conclude: For metrizable spaces, the covering dimension is the same as the least uniform dimension in any metric uniformity compatible with the topology. I do not know whether the word "metric" can be omitted.

\section{REFERENCES}

1. M. Atsuji, Uniform continuity of continuous functions of metric spaces, Pacific J. Math. 8 (1958), 11-16.

2. K. Borsuk, On an irreducible 2-dimensional absolute retract, Fund. Math. 37 (1950), 137-160.

3. C. Dowker, Mapping theorems for non-compact spaces, Amer. J. Math. 69 (1947), 200242.

4. C. Dowker and W. Hurewicz, Dimension of metric spaces, Fund. Math. 43 (1956), 8388.

5. S. Ginsburg and J. Isbell Some operators on uniform spaces, Trans. Amer. Math. Soc., to appear.

6. J. Isbell, Zero-dimensional spaces, Tohoku Math. J. 7 (1955), 1-8.

7. Isbell, Euclidean and weak uniformities, Pacific J. Math. 8 (1958), 67-86. 
8. Katetov, On real-valued functions in topological spáces, Fund. Math. 38 (1951), 85-91.

9. Yu. Smirnov, On she dimension of proximity spaces, Mat. Sb. 38 (1956), 283-302.

10. Yu. Smirnov, Geometry of infinite uniform complexes and $\delta$-dimension of proximity spaces, Mat. Sb. 40 (1956), 137-156.

UNIVERSITY OF WASHINGTON 



\section{PACIFIC JOURNAL OF MATHEMATICS}

\section{EDITORS}

\section{David Gilbarg}

Stanford University

Stanford, California

R. A. Beaumont

University of Washington

Seattle 5 , Washington
A. L. Whiteman

University of Southern California

Los Angeles 7, California

L. J. PAIGE

University of California

Los Angeles 24, California

\section{ASSOCIATE EDITORS}
E. F. BECKENBACH
C. E. BURGESS
E. HEWITT
A. HORN

\author{
V. GANAPATHY IYER \\ R. D. JAMES \\ M. S. KNEBELMAN \\ L. NACHBIN
}
I. NIVEN
E. G. STRAUS
T. G. OSTROM
H. L. ROYDEN
G. SZEKERES
M. M. SCHIFFER
F. WOLF
K. YOSIDA

\section{SUPPORTING INSTITUTIONS}

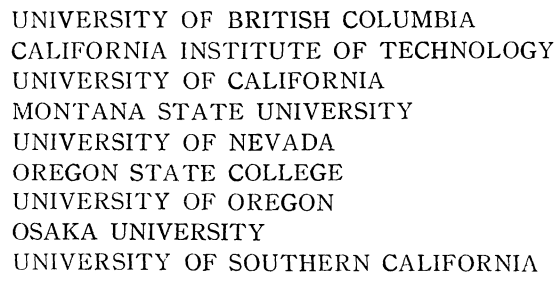

UNIVERSITY OF BRITISH COLUMBIA CALIFORNIA INSTITUTE OF TECHNOLOGY UNIVERSITY OF CALIFORNIA MONTANA STATE UNIVERSITY UNIVERSITY OF NEVADA OREGON STATE COLLEGE UNIVERSITY OF OREGON OSAKA UNIVERSITY UNIVERSITY OF SOUTHERN CALIFORNIA

STANFORD UNIVERSITY

UNIVERSITY OF TOKYO

UNIVERSITY OF UTAH

WASHINGTON STATE COLLEGE

UNIVERSITY OF WASHINGTON

AMERICAN MATHEMATICAL SOCIETY CALIFORNIA RESEARCH CORPORATION HUGHES AIRCRAFT COMPANY SPACE TECHNOLOGY LABORATORIES

Printed in Japan by Kokusai Bunken Insatsusha (International Academic Printing Co., Ltd.), Tokyo, Japan 


\section{Pacific Journal of Mathematics}

\section{Vol. 9, No. 1 \\ May, 1959}

Julius Rubin Blum and Murray Rosenblatt, On the structure of infinitely

divisible distributions . ............................. 1

Robert Geroge Buschman, Asymptotic expressions for

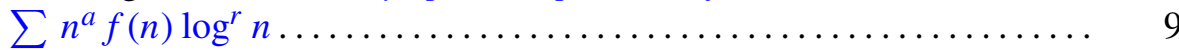

Eckford Cohen, A class of residue systems $(\bmod r)$ and related arithmetical

functions. I. A generalization of Möbius inversion .............. 13

Paul F. Conrad, Non-abelian ordered groups ................... 25

Richard Henry Crowell, On the van Kampen theorem............... 43

Irving Leonard Glicksberg, Convolution semigroups of measures ........ 51

Seymour Goldberg, Linear operators and their conjugates ............ 69

Olof Hanner, Mean play of sums of positional games .............. 81

Erhard Heinz, On one-to-one harmonic mappings ................ 101

John Rolfe Isbell, On finite-dimensional uniform spaces . . ........... 107

Erwin Kreyszig and John Todd, On the radius of univalence of the function

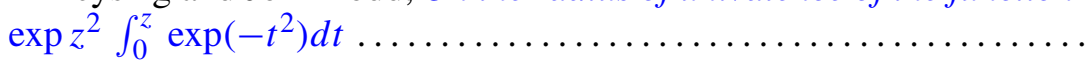

Roger Conant Lyndon, An interpolation theorem in the predicate

calculus......................................... 129

Roger Conant Lyndon, Properties preserved under homomorphism ........ 143

Roger Conant Lyndon, Properties preserved in subdirect products ....... 155

Robert Osserman, A lemma on analytic curves ................ 165

R. S. Phillips, On a theorem due to Sz.-Nagy..................... 169

Richard Scott Pierce, A generalization of atomic Boolean algebras ....... 175

J. B. Roberts, Analytic continuation of meromorphic functions in valued fields................................. 183

Walter Rudin, Idempotent measures on Abelian groups ................ 195

M. Schiffer, Fredholm eigen values of multiply-connected domains ........ 211

V. N. Singh, A note on the computation of Alder's polynomials ......... 271

Maurice Sion, On integration of 1-forms ...................... 277

Elbert A. Walker, Subdirect sums and infinite Abelian groups........... 287

John W. Woll, Homogeneous stochastic processes . .................. 293 